\title{
PRAGMÁTICA Y RELACIÓN INTRATEXTUAL: El caso de hasta, incluso y ni siquiera
}

\author{
CATALINA FUENTES RODRÍGUEZ \\ (Universidad de Sevilla)
}

0. Montones de páginas se han llenado intentando poner orden en la cuestión del adverbio para siempre terminar con la idea de que en dicha categoría se agrupan multitud de elementos de comportamiento muy distinto. En consecuencia, actualmente en la bibliografía al uso, se estudian algunos de ellos por separado y no se concede excesiva importancia a su encasillamiento.

De toda esta preocupación y del interés por sugerir "otro" análisis ha surgido el presente estudio. Pensamos que podría hacerse una aproximación al comportamiento funcional de estos elementos, y queremos, al mismo tiempo, dar cuenta de algunos intentos hechos en otros idomas.

Partimos de la observación de S. Greenbaum (1970) que los divide en «adjuncts, conjuncts and disjuncts" y establece una serie de pruebas para distinguir su valor. Nuestra traducción será adjuntos, enlaces conjuntivos y disjuntos. Elegimos el nombre de enlaces conjuntivos porque creemos que se adapta mejor a su comportamiento textual. Nos vamos a quedar en el análisis de los primeros, ejemplificándolo en dos términos españoles que tienen un uso muy peculiar. Al mismo tiempo intentamos aplicarles un análisis que muchos autores de la lingüística angloamericana han empleado y que Bosque (1980) recoge: situarnos en un punto de vista pragmático, utilizando un análisis presuposicional. Quede, pues, como una puesta a punto de nuevas metodologías sobre el adverbio que, quizás, se ajusten mejor a su valor lingüístico.

1. Greenbaum estableció un test a base de 10 criterios, que podriamos traducir de la siguiente manera:

1. Inaceptable en posición inicial de cláusula.

2. Inaceptable en posición inicial si la cláusula es negativa.

3. Inaceptable en posición inicial si está en una unidad tonal independiente. 
4. Puede ser foco de una interrogación. Para ello podrá ser el elemento enfatizado en una interrogación alternativa.

5. Puede ser foco de una negación. Para ello también podrá contrastarse en una negación alternativa.

6. Puede ser rematizado por only.

7. Puede servir de foco en una «cleft-sentence».

8. Puede servir como respuesta a una transformación interrogativa de la oración, introducida por un adverbio interrogativo.

9. Puede ser afectado por How en una interrogación o exclamación (por un adverbio exclamativo o interrogativo).

10. Puede ser el foco de una expresión comparativa: más...que (Greenbaum, 1970: 18-23).

Según él, los elementos que cumplen los 3 primeros criterios, o bien el 4 o bien el 5 son «adjuntos». Los que no cumplen ninguno de los 5 pueden ser «enlaces conjuntivos" o "disjuntos" (Rojas Nieto, 1982: 34-35): "disjuncts can serve as a response to a yes-no question, though sometimes they require to be accompanied by yes or no; conjuncts cannot serve as a response to a yes-no question even if accompanied by yes or no" (Greenbaum, 1970: 25).

$Y$, aparte, los disjuntos indican una evaluación de lo que se dice, con respecto a la forma de la comunicación o a su contenido, mientras que los enlaces conjuntivos indican una conexión con lo que ha venido antes. Ilustremos esto con ejemplos:

$11 a /:$ «Jesús trabaja bien»

|2a/: «Afortunadamente, está equivocado»

/3a/: «Sin embargo, vendrá»

Apliquemos el test:

1. Ninguno de ellos lo cumple, ya que el elemento 1 podemos tenerlo asi: $/ 1 \mathrm{~b} /$ : «Bien trabaja Jesús»

2. Los tres pueden ir en oraciones negativas:

$11 \mathrm{c} /$ : «Jesús no trabaja bien»

/2b/: «Afortunadamente, no está equivocado"

$\mid 3 \mathrm{~b} /:$ : Sin embargo, no vendrá»

3. El elemento bien si cumple la prueba, ya que no puede ir en posición tonal independiente:

*/1d/: «Bien, Jesús trabaja»,

ya que se genera un valor distinto. Bien pasaría a ser una muletilla, un adverbio de asentimiento en todo caso, pero no un incidente en el verbo que sigue.

Los otros dos elementos sí están aislados entonativamente del resto de la oración, como puede verse en los ejemplos anteriores.

4. De nuevo bien puede cumplirla, ya que puede ser foco en una interrogación: 1 e/: «Jesús trabaja bien o mal?»

Pero no los otros dos:

*/2c/: « ¿Está equivocado afortunadamente o desgraciadamente?»

*/3c/: «¿Vendrá sin embargo o además?»

5. En este ocurre igual. El ejemplo 1 sí lo cumple:

/1f/: «Jesús no trabaja bien sino ma/»

Pero no los demás: 
*/2d/: «No está equivocado afortunadamente sino desgraciadamente»

*/3d/: «No vendrá sin embargo sino además"

6. Esta prueba no parece funcionar en español:

*/1g/: «Sólo bien trabaja Jesús»

*/2e/: "Sólo afortunadamente está equivocado»

*/3e/: "Sólo sin embargo vendrá»

7. El número 1 podría cumplirla:

/1h/: «Bien es como trabaja Jesús»

Pero no los otros:

*/2f/: «Afortunadamente es como está equivocado»

*/3f/: «Sin embargo es como vendrá»"

8. En esta otra ocurre igual. Bien puede satisfacer la prueba, puesto que es un elemento que tiene contenido designativo completo:

/1i/: «¿Cómo trabaja Jesús? Bien"

Pero no así los otros dos:

*/2g/: «¿Cómo está equivocado? Afortunadamente»

*/3g/: «¿Cómo vendrá? Sin embargo»

9. De nuevo el número 1 puede cumplirlo, frente a los otros dos casos:

/1j/: «Qué bien trabaja Jesús!»

*/2h/: «iQué afortunadamente está equivocado!»

*/3h/: «iQué sin embargo vendrá!"

10. Esta prueba arroja el mismo resultado:

$11 \mathrm{k} /$ : «Jesús trabaja más bien (mejor) que Juan"

*/2i/: «Está equivocado más afortunadamente que tú"

*/3i/: "Vendrá más sin embargo que tú"

Por lo tanto, los perfiles serían los siguientes:

bien afortunadamente sin embargo

\begin{tabular}{rrrr}
\hline 1. & - & - & - \\
2. & - & - & - \\
3. & + & - & - \\
4. & + & - & - \\
5. & + & - & - \\
6. & - & - & - \\
7. & + & - & - \\
8. & + & - & - \\
9. & + & - &
\end{tabular}

Bien sería, pues, adjunto. Afortunadamente, un disjunto, ya que indica una evaluación sobre lo que dice. $Y$ puede ser respuesta con si y no:

$12 \mathrm{j} /$ : « ¿Está equivocado? Afortunadamente, sí». Esto lo rechazan Allerton y Cruttenden porque no se cumple en todos (Allerton-Cruttenden, 1974).

Pero no sin embargo:

*/3j/: « ¿Vendrá? Sin embargo, Sí» 
Además, este elemento indica una oposición con respecto al enunciado anterior, que podría ser:

$13 \mathrm{k} /$ : «No lo he invitado. Sin embargo, vendrá»

Es un enlace conjuntivo, pues.

Como podemos ver, se distinguen de esta manera aquellos adverbios que van modificando a un constituyente de la oración, y que tienen valor léxico completo (=adjuntos), de aquellos otros, llamados también adverbios o atributos oracionales, que se sitúan al margen de la oración (=disjuntos y enlaces conjuntivos). (Cfr. Alarcos Llorach, 1984: 312, que se refiere sobre todo a los disjuntos. Y Rojas Nieto, 1982: 34, que lo extiende a todos. Esto puede ser discutible. Sólo puede ser admitido refiriéndose a la posición, no a su funcionamiento.)

Estos elementos no han sido objeto de un estudio organizado desde esta perspectiva. Sí hay algunos parciales. Asi, los disjuntos son los que se traducen como «adverbios de frase» (cfr. Schreiber, 1971; Martin, 1974 y Dominguez de RodríguezPasqués, 1970), y los enlaces conjuntivos se han incluido tradicionalmente con las conjunciones coordinadas, bien como una conjunción más (cfr. la mayoría de las gramáticas tradicionales), o como un refuerzo de ellas (Echaide, 1974, para la adversativa).

Los adjuntos son los considerados modificadores de «la significación del verbo o de la del adjetivo, y a veces la de otro adverbio» (Martínez Amador, 1966: 92), si bien esta no es su única función. Así hay algunos adjuntos que parecen modificar a un sintagma nominal. La gramática los ha tratado a veces como preposiciones (Alcina-Blecua, 1983), o adjetivaciones (Carratalá, 1980). Así lo hace, por ejemplo, $M$. Seco con respecto a incluso: «Puede funcionar como preposición: «Todo me parecia bien, incluso la persona de doña Javiera (Galdós, El amigo Manso, 279)» (Seco, 1967: 197).

Sin embargo, en un uso equivalente, hasta es considerado «adverbio de cantidad, equivalente a incluso, aun: gritó, lloró y hasta pataleó; "iy qué esquiva para tus bienes / y qué amarga hasta cuando amél» (Gabriela Mistral, Desolación, 33)" (Seco, 1967: 188).

No obstante, esos elementos veremos que corresponden perfectamente a los adjuntos. Nosotros nos vamos a centrar en unos que ofrecen unas características especiales: hasta, incluso y ni siquiera. Su estudio revelará si es necesario ampliar las características funcionales del adverbio, dividirlo en varias categorías o únicamente cambiar de método de análisis.

2. Antes que nada hay que remitir a los muchos estudios que hay sobre el equivalente inglés de los dos primeros, sobre todo: even. Asi, véase Fraser (1971), Anderson (1972), Shanon (1978), Horn (1969)...

El primero de ellos plantea ya uno de los problemas que van a preocupar más en la gramática generativa: dónde se introduce even, en la estructura de superficie o antes, cosa que no quedaba muy clara. En su interpretación toca una serie de puntos, unos más aprovechables por nosotros que otros.

Uno de ellos es la aparición de even más de una vez en la oración, que él admite. Anderson se opondrá a esto ya que por sus características semánticas sólo puede aparecer una vez. Para probarlo usa un análisis presuposicional más completo qui- 
zás que el que Horn establece, y de él surgen las incompatibilidades. Horn lo analiza en términos de Aserción y Presuposición, pero no termina por precisar el valor de violación de lo esperado, de sorpresa, que aportan estos elementos. Su esquema, que luego Bosque tomará, es:

«(54) Even ( $x=a, F x)$

$P:(\exists y)(y \neq x \& F y)$

A: FX (Horn, 1969: 105).

Deducimos, pues, que al hablar de características semánticas se refiere a las implicaciones pragmáticas que llevan. En español es posible que haya ejemplos con dos ocurrencias, ya que el even inglés puede traducirse por hasta e incluso, elementos de funcionamiento distinto.

Aparte de esto, un punto fundamental en la interpretación de Anderson y en la de muchos otros autores (ya volveremos a ello) es el énfasis, que le sirve para delimitar el elemento al que modifica. En ese sentido, veremos, lo llama H. Contreras (1978: 85) rematizador y $\mathrm{R}$. Cano (1982) lo aceptará matizando esto: lo que enfatiza o rematiza es la función semántica: el que el elemento $X$ sea agente, instrumento de la acción...: «lo realzado no es exactamente lo referido por ese sintagma nominal, sino el hecho de que se encuentre en la situación definida por la función sintáctica que desempeña" (Cano, 1982: 232). Y así "el valor semántico de hasta no es el de señalar los elementos 'nuevos' de un enunciado, sino el 'énfasis' (...) con que se presenta su función semántica en la frase» (Cano, 1982: 234).

La relación de hasta (y de incluso y ni siquiera, añadimos nosotros) y el énfasis es estrecha, ya que las presuposiciones provocan el que ese elemento resulte sorprendente en cierto modo. Se recalca, pues, sobre el resto de la oración. Esto está relacionado, según Anderson, con la posición: even domina al elemento con énfasis aunque vaya alejado sintácticamente de él. Nosotros tenemos nuestras dudas con respecto a esto. En un ejemplo del tipo:

14/: "Yo hasta le ofreci cerillas",

pienso que incide sobre todo el sintagma verbal, aunque el elemento más sorprendente sea el implemento.

Así R. Cano señala normas sobre su ámbito de aplicación: cuando va con un sintagma nominal le afecta a él solo. Pero cuando precede a un verbo, «la aplicación de hasta no se realiza sólo sobre el verbo, sino también sobre los elementos que le siguen, y en concreto sobre todo el sintagma verbal o predicativo (excluyendo los 'adjuntos' locativos o temporales)" (Cano, 1982: 234). Pero, al mismo tiempo, añade que en estos casos, además del énfasis «parece tener mayor pertinencia el concepto de 'nuevo' o 'rema'» (Cano, 1982: 234), cosa que dependerá del contexto de cada oración.

Por último, Anderson señala que con verbos modales el comportamiento de even es paralelo al de la negación, pero no lo precisa. Digamos nosotros que hasta puede modificar al verbo modal o al verbo de la oración subordinada:

15a/: "Hasta creo que vendré al concierto".

$15 \mathrm{~b} /$ : "Creo que hasta vendré al concierto".

Finalmente, B. Shanon lo estudia junto con only, almost y hardly. Pretende caracterizarlos por separado y luego como grupo. Sigue en la línea de los autores anteriores: su análisis también se basa en la presuposición de una escala a la que 
pertenece el elemento introducido por even o alguno de los otros. Para él son cuatro operadores escalares. Para su análisis señala dos puntos:

S 1-condiciones iniciales o lo esperado.

S 2-situación en cuestión.

En even $S 2$ está más alto que $S 1$ - supera lo esperado y como consecuencia se producirá esa sorpresa de que hablan algunos autores.

Sin embargo, su análisis se restringe a los adjetivos de altura, donde se aplica mejor. De ahí que los puntos que establece no nos interesen ni sirvan para ningún otro elemento. Pero, como hemos podido observar, el estudio no es sólo de comportamiento distribucional, sino también pragmático. El contenido de estos términos se basa en presuposiciones que el hablante y el oyente poseen y lo condicionan a la hora de elegir un elemento u otro. Se gira, pues, hacia la pragmática, ciencia joven que tiene mucho que aportar en sintaxis.

3. Todos estos estudios han quedado recogidos para el español en I. Bosque, pero este autor, que pone a punto la cuestión, se centra únicamente en incluso-ni siquiera y su consideración de términos de polaridad positiva y negativa (Bosque, 1980: 113-121). El punto a discutir en esa parte de su libro es si ni siquiera se genera por transporte de la negación, como decía M. L. Rivero (1977: 109) de inc/uso no o por concordancia negativa, como parece concluir Bosque.

Nosotros, por el contrario, pensamos que la oposición debe establecerse entre dos polos: uno positivo ocupado por hasta e incluso (con sus diferencias), y otro por $n i$ (no) siquiera, que también tiene sus variantes formales como veremos. Es una pareja funcional que puede tener formas distintas de expresión. El contenido de estos elementos se basa en un esquema presuposicional ya establecido por Horn y Anderson, y que luego otros autores, como Fauconnier (1975 a y b), critican, añadiendo que no da perfecta cuenta del valor de even.

Este autor, por su parte, propugna que lo que presupone even es una escala pragmática en la que el elemento introducido por él se coloca en uno de los extremos. Lo representa así:

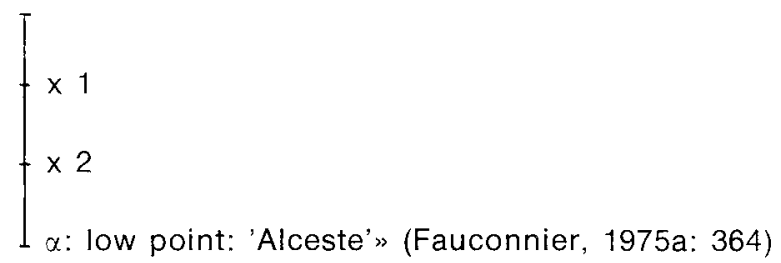

para la frase: “(110) Even Alceste came to the party» (Fauconnier, 1975a: 364).

El principio escalar en general queda expresado de la siguiente manera:

«In general, then, pragmatic scales are associated with proposition schemata (...)

Such propositional schemata have the general form $R(x, \ldots)$. (The dots stand for possible additional free variables such as $y$ in $x$ bothers $y$.) If $x 2$ is lower than $x 1$ on the scale $S$ associated with $R(x, \ldots)$, then $R(x 2, \ldots)$ implies $R(x 1, \ldots)$; thus, in particular if $R$ holds for the lowest element on $S$, it holds for all elements of $S$ (call this the scale principle)» (Fauconnier, 1975a: 362).

Por lo tanto, el contenido de 
/6a/: «Hasta Antonio tenía miedo»

es:

a) Afirmación: Antonio tenía miedo.

b) Presuposición: Todos los demás elementos que pertenecen a su mismo paradigma también tenían miedo. Antonio está en el punto más alto. Nuestra presentación es distinta a la de Fauconnier, va en sentido ascendente, no de probabilidad de aparición, como parece ser la de este autor.

c) Por lo tanto, era el que menos esperábamos que tuviera miedo. Es sorprendente, pues. Es, como dice Bosque refiriéndose a Fauconnier: «el extremo de una hipotética escala de probabilidad» (Bosque, 1980: 118).

Por lo tanto, el valor de «límite de movimiento» original de la preposición locativa, se estiliza hasta entenderse en el valor de «límite», esta vez «de lo que se puede creer, decir u opinar» (Cano, 1982: 230). Es decir, el punto más alto de una escala.

Pero esta escala hay que entenderla en el sentido en que lo han propuesto sus autores. Es decir, se trata de una ordenación pragmática de posibilidades. Desde el punto de vista de la praxis el hablante considera que para el oyente lo que él le va a decir es lo menos esperado para él, lo más sorprendente. Supone, pues, que en la mente del oyente y según el contexto hay una escala de cosas más probables y otras menos. En este sentido, la dicha es la menos probable y ocupa el lugar más bajo (o el lugar más alto, por ser la más sorprendente). $E$ indirectamente si se da la menos probable se incluyen, por una implicatura conversatoria, todas las demás. Pero de nuevo advertimos que no se trata de que los hechos estén en escala, sino que para el hablante se supone una jerarquía de lo más esperado a lo menos esperado.

Como esto podría plantear problemas y equívocos, nuestro análisis no hará hincapié de ahora en adelante en esta idea. La formulación que daremos para estos elementos será la siguiente:

a) Afirmación: $X$.

b) Presuposición: _ Otros elementos del mismo paradigma de $X$ se dan.

- El hablante supone que el oyente no esperaba el hecho enunciado. Y provoca sorpresa, por tanto.

Si lo tuviéramos con ni siquiera el esquema no variaría sustancialmente:

/6b/: "Ni siqueira Antonio tenía miedo».

a) Afirmación: Antonio no tenía miedo.

b) Presuposición: Todos los demás elementos de la serie tampoco tenían miedo.

c) Antonio es el elemento que más probablemente esperábamos que tuviera miedo. Es el que se pensaba que no cumpliría esa predicación. Es, pues, sorprendente que no la cumpla.

Si observamos, se trata de convertir en negativo tanto afirmación como presuposición. Aquí sería:

a) Afirmación: $-X$.

b) Presuposición: Otros elementos del misıno paradigma también son negados. No se esperaba $-X$, sino todo lo contrario: $X$. De ahí la sorpresa.

El contenido de los dos elementos es el mismo. Se trata de afirmar un miembro y suponer que todos los que pertenecen a su misma serie también lo cumplen, sea el juicio afirmativo o negativo. $Y$ una segunda presuposición estaría en que el hablante piensa que para el oyente esta aserción no era esperada y le ha producido asombro. 
De ahí que estudiemos estos dos miembros como componentes polares de una pareja.

No estamos, pues, de cuerdo con I. Bosque cuando dice, refiriéndose a inc/uso, que tiene el mismo análisis presuposicional de hasta (ya veremos la distinción entre ambos): «Las oraciones de $n i$ siquiera alteran radicalmente el conjunto de presuposiciones que hay que asociar al sintagma que cuantifica al adverbio incluso (polaridad contextual). El adverbio al menos, por el contrario, no está sujeto a tal alteración" (Bosque, 1980: 120). Sin embargo, desde nuestro punto de vista no cabe admitirse esto, ya que el análisis de una oración con al menos:

$/ 6 \mathrm{c} /$ : «Al menos Antonio tenía miedo» sería:

a) Afirmación: Antonio tenía miedo.

b) Presuposición: Algunos otros no tenían miedo. Los que conozco no tenían miedo. No tengo noticias de algún otro que tuviera miedo.

c) Es el único elemento de su paradigma que se da. El hablante se alegra de que haya un elemento que lo cumpla. Esperaba y deseaba que hubiera uno que lo cumpliera.

Por lo tanto, no incluye a los demás elementos. No provoca sorpresa, sino que sugiere un deseo del hablante, ahora cumplido. El problema, como vamos viendo, se complica al mezclarse varios elementos que son cuasi-sinónimos, o que alternan con él.

Nuestro punto de partida es, pues, según el análisis presuposicional, que creemos más adecuado, hasta (o incluso) se opone a ni siquiera en base a:

Positivo / negativo.

4. Estos elementos se comportan como verdaderos adjuntos. Si les aplicamos el esquema de Greenbaum tendremos:

1. Todos pueden aparecer en inicial, como vemos por los ejemplos.

2. El primero, hasta, es imposible que aparezca en una oración negativa:

*/7a/: "Hasta con ella no se enfada" mientras que ni siquiera siempre va en oraciones negativas:

$17 \mathrm{~b} /$ : "Ni siquiera con ella se enfada»

Pero eso se refiere a las negativas formales, no a las que lo son por el significado. Asi es aceptable una oración del tipo:

/8/: «Hasta dejó de venir por casa".

Con incluso, sin embargo, sí puede aparecer una negación:

19/: "Incluso no se asustó cuando se lo contó»"

3. Ninguno de los dos primeros pueden aparecer como unidad entonativa independiente:

*/10/: «Hasta, por las tardes sale»

*/11/: «Ni siquiera, voy al Rocío».

Ya esta prueba nos demuestra que son adjuntos. Ninguno de ellos puede tener ningún empleo como enlace conjuntivo o disjunto. Pero con incluso pueden darse las dos posibilidades. Puede aparecer al igual que hasta, aunque con una cierta movilidad:

/12a/: «Ha perdido incluso el sueño» 
$/ 12 \mathrm{~b} /$ : «Ha perdido el sueño, incluso».

En este segundo caso va aislado fonéticamente del elemento al que acompaña. $O$ bien puede aparecer como margen oracional en su empleo como enlace conjuntivo:

113/: «En realidad, mi mayor, yo creí que el cadete Arana era culpable, al menos en parte, por estar mal emplazado, por haber demorado en el salto. Incluso, hasta podía pensarse que la bala salió de su propio fusil» (M. Vargas Llosa: La ciudad y los perros, 318).

Sin embargo, en las dos siguientes pruebas varian: son negativas para todos. Ninguno de ellos puede ser contrastado en una interrogación (4), o en una negación (5). Veámoslo con respecto a las oraciones:

/14a/: «Comimos hasta fresas"

$/ 14 \mathrm{~b} /$ : «No comimos ni siquiera fresas"

$114 \mathrm{c} /$ : «Comimos incluso fresas»

La prueba 4 nos daría:

?/14d/: «¿Comísteis hasta fresas o sólo fresas?"

?/146/: «¿No comísteis ni siquiera fresas o sólo fresas?"

?/14f/: « ¿Comísteis incluso fresas o sólo fresas?» Y la 5:

?/14g/: «No comimos hasta fresas sino sólo fresas"

$? / 14 \mathrm{~h} /$ : «No comimos ni siquiera fresas sino sólo fresas"

?/14i/: "No comimos incluso fresas sino sólo fresas".

En estos ejemplos lo que se contrapone es todo el sintagma al que afectan hasta, ni siquiera o incluso, con el presentado por sólo.

Por lo tanto, entre los adjuntos habría que distinguir entre aquellas unidades que están más ligadas a un determinado elemento al que introducen y afectan: así hasta, y otros... Y otras que son más libres y admiten movilidad, de manera que pueden ir pospuestas o antepuestas al elemento sobre el que inciden: así los llamados deicticos, o por ej., incluso. Y también ni siquiera, que puede aparecer detrás del elemento, como vamos a ver:

/15a/: «Había leído incluso relatos de ciencia-ficción»

$115 \mathrm{~b} /$ : «Había leído relatos de ciencia-ficción incluso"

$115 \mathrm{c} /$ : "No había leído relatos de ciencia-ficción siquiera".

El elemento afectado por incluso será el que forme grupo fónico con él. En /15a/ es el implemento. En /15b/es fácil, ya que va al final.

Al mismo tiempo, esto nos sirve para distnguir, junto con algunas de las pruebas restantes, entre aquellos elementos que tienen contenido léxico pleno, del tipo de bien, y los que fundamentan su valor en un contenido presuposicional o pragrá́tico, del tipo de los que analizamos.

6. Es negativa en todos, ya que sólo no es un rematizador en español:

*/14j/: «Sólo hasta comimos fresas».

En todo caso, todo el segmento funcional que introduce hasta:

*/14k/: «Sólo hasta fresas comimos»,

pero tampoco es muy frecuente.

*/14i/: «Sólo ni siquiera fresas comimos"

*/14m/: «Sólo incluso fresas comimos»

7. Tampoco pueden aparecer destacados por es...que: 
?/14n/: "Hasta fresas es lo que comimos" Aceptable en todo caso siendo el segmento entero el que es puesto de relieve, no el elemento que estudiamos. Igual con ni siquiera:

$? / 14 \tilde{n} /:$ «Ni siquiera fresas es lo que comimos»

?/140/: "Incluso fresas es lo que comimos".

8. No pueden servir como respuesta a una transformación interrogativa, ya que no llevan contenido lexemático pleno:

*/14p/: «¿Comísteis fresas? Hasta»

*/14q/: «¿Comisteis fresas? Ni siquiera»

*/14r/: «¿Comisteis fresas? Incluso».

9. No cabe tampoco la posibilidad de ser modificado por un adverbio exclamativo:

*/14s/: «jCuánto hasta comimos fresas!»

*/14t/: « ¿Cómo ni siquiera comimos fresas!»

*/14u/: «iCómo incluso comimos fresas!»

10. Ni pueden ser el elemento que se somete a una comparación:

*/14v/: «Comimos fesas más hasta que además"

*/14x/: "No comimos fresas más ni siquiera que incluso"

*/14y/: “Comimos fresas más incluso que ni siquiera».

Son, pues, adjuntos, aunque con ciertas diferencias. Hasta e incluso se distinguen, aparte de porque este último puede ser enlace conjuntivo, en la no movilidad del primero frente a la movilidad del segundo. Hasta debe preceder al elemento al que se refiere siempre, mientras incluso puede precederlo o ir después, como vimos. Además, este último puede ir con oraciones negativas, algo imposible para hasta. No es, por lo tanto, un elemento polar. Entonces pueden tomarse como dos variantes formales para un mismo valor funcional, aunque viendo sus diferencias y sabiendo que incluso es un elemento que tiene otras dimensiones.

También en el polo negativo hay variaciones formales. Vimos que no tenía por qué preceder siempre ni siquiera, ya que puede englobar al sintagma al que se refiere:

116/: «Ni Juan siquiera lo creía»

117/: «Lo que está lejos de nosotros a lo mejor ni existe siquiera» (C. J. Cela: Mazurca para dos muertos, 218).

118/: «No lo vi siquiera"

Es decir, el elemento negativo selecciona ni la mayoría de las ocasiones, pero cuando aparece englobando a un verbo el elemento negativo puede variar a no. El elemento negativo se formularía así: Negación+siquiera.

Pero el conjunto formado por el adverbio + su elemento si disponen de movilidad dentro de la oración. Pueden ir en posición inicial, más usada porque así se recalca su valor rematizador, pero también puede adoptar otras posiciones:

119/: "Cuando me siento cansada, me molesta hasta la televisión»

120/: "No me decido a cambiarme de piso ni siquiera porque llueve»

121/: "Me gusta, incluso cuando llueve, sentarme en la terraza"

Podríamos decir entonces que los verdaderos elementos polares son hasta y $n i$ siquiera, ya que son los que manifiestan restricciones con la negación-afirmación. Lo que ocurre es que hay otros elementos que pueden aportar su mismo valor. Así, otro elemento que puede usarse en lugar de hasta es aun: 
122/: "Aun sin comer iba a trabajar»

La diferencia entre esos tres elementos afirmativos, según R. Cano, es la siguiente:

Hasta I Aun / Incluso

'límite enfático' / 'concesivo' / 'integración en una serie' (Cano, 1982: 244).

Estas son las ideas predominantes en cada uno, aunque pueden tener las tres. Por lo tanto, siempre según este autor, sería el uso el factor distintivo: hasta e incluso se usan en todos los sectores, pero el primero es más popular.

Para nosotros la diferencia es de funcionamiento: hasta y aun no pueden ser enlaces conjuntivos, y preceden siempre al elemento al que afectan. Inc/uso sí es enlace, y puede ir pospuesto a dicho elemento. Este último no es término de polaridad positiva, frente a hasta que sí lo es. Aun está a medio camino. No se admite la combinatoria con el adverbio negativo no, sino que debe convertirse en ni aun oni siquiera:

*/23a/: «Aun para comer no viene»

123b/: "Ni aun para comer viene"

123c/: "Ni siquiera para comer viene"

Al ser un elemento átono aun no se usa con la misma frecuencia que hasta.

Ni siquiera también tiene elementos equivalentes o variantes contextuales, como ya hemos ido diciendo. Así, por ejemplo, ya Bosque se refería al uso de ni aun o ni, por este elemento. Para Martínez Amador el elemento que estudiamos, analizado por él como una combinación de siquiera tras ni, equivale a ni aun, aunque según Bello, al que cita, no completamente. Nos dice: «Observa el insigne gramático que a su juicio ni aun se aplica a gradaciones tácitas, tanto de menos a más, como de más a menos" (Martínez Amador, 1966: 1.352). Y lo cita a continuación para ver su diferencia con ni siquiera: "Si no me engaño, dice, sólo para la segunda especie de gradaciones es propio ni siquiera'» (Martínez Amador, 1966: 1.352).

Para Bosque, sin embargo, no se trata de eso, ya que pueden alternar en los ejemplos, sino de una característica de combinación sintáctica: ni aun puede ir sólo con sintagmas nominales; ni siquiera con sintagmas nominales y verbales:

124a/: «No llegamos ni siquiera a Roma»

$124 \mathrm{~b} /$ : "No llegamos ni aun a Roma»

125a/: "Ni siquiera hablamos durante el camino"

*/25b/: «Ni aun hablamos durante el camino»

Pero ni siquiera puede reducirse a alguno de sus componentes según el contexto. Así puede alternar con ni:

/26/: «-... Me odia.

- ¿A ti? Ni sabe cómo te apellidas" (M. Vargas Llosa: La ciudad y los perros, 220).

127/: «Sublime estribación de unas alturas

sin nombre conocido; lo allí escrito

escrito quedará ante las estrellas.

$\mathrm{Ni}$ sé lo que he vivido. La memoria

...» (F. Rodriguez-Izquierdo: Recinto en la palabra, 39-40).

128/: «Ni su secretaria se escapó de su oleada de violencia».

C. Rojas Nieto considera este elemento, junto con otros como sino, pero..., coordinantes de miembros no equivalentes, que no tienen su función nexual habitual, sino 
que toman en el contexto una carga léxica distinta. $Y$, con respecto a ni y sino, «presuponen un absoluto negativo correspondiente al elemento que introducen.

No sabia ni escribir = No sabía nada, ni escribir.

No tomaba carne ni cuando no había otra cosa = No tomaba carne nunca, ni cuando..." (Rojas Nieto, 1982: 243-244).

Ya A. M. Barrenechea (1979: 27, n. 14) reconocía que tenían función adverbial, función que Rojas Nieto interpreta como «paralela a la de los adverbios rematizadores (sólo, hasta) a que hace mención Heles Contreras (...), que, además, incluye entre éstos, la expresión ni siquiera (Rojas Nieto, 1982: 235, n. 176).

Nosotros también nos adherimos a esto, si bien no consideramos que se trate de elisión de ningún cuantificador absoluto, sino que presuponen toda una serie de elementos que cumple lo afirmado o negado. Se trata de valor presuposicional, no de elemento elidido.

También alterna con siquiera, que para M. Moliner es una expresión exhaustiva como la forma plena. Por expresión exhaustiva entiende "ciertas expresiones conjuntivas, «ni» y otras formadas con «ni», que denotan la absoluta carencia de la cosa que se expresa» (Moliner, 1983: 1.253). También puede tener, nos señala, en lenguaje vulgar, la forma ni tan siquiera.

Siquiera sólo puede aparecer como reducción de ni siquiera tras un activador negativo:

/29a/: «No hice ni siquiera pasteles»

129b/: «No hice pasteles siquiera», o

129c/: «No hice siquiera pasteles».

Por concordancia negativa se elimina ni. También con otro activador negativo:

$129 \mathrm{~d} /:$ :Sin haberlo hecho siquiera".

Pero no cuando ni siquiera aparece en cabeza de la frase, ya que elimina la presencia de no:

129e/: "Ni siquiera pasteles hice»

?/29f/: «Siquiera pasteles hice».

Aquí tiene un valor distinto: equivale a al menos.

Con este elemento lo relaciona l. Bosque, diciéndonos que siquiera es un TPN cuyo TPP es al menos. Y que puede usarse en contextos irreales. Comprobemos esta afirmación:

130/: "Ven siquiera a darme un beso".

Vuelve a equivaler a al menos. Y como hemos visto en /29f/, es afirmativa, con to cual no puede decirse que sea un TPN.

Habria, pues, que matizar. Según nuestra investigación, hay dos valores de siquiera (tomado como adverbio de cantidad o modal, como dice la R.A.E. Aparte de esto puede ser distributivo, un adversativo o una conjunción seguida de sea, fuese... Cfr. R.A.E., 1984: 1.249):

- en contextos en que precede una negación equivale a ni siquiera. Por redundancia de ni...no (u otro activador negativo) se elimina.

- en contextos afirmativos, o con una negación que le siga, equivale a al menos:

?/31/: «Siquiera café no tomamos» (=al menos)

Es, pues, un elemento que alterna con uno o con otro, una variante contextual de alguno de los dos. 
Por lo tanto, las variantes de los elementos de la oposición serían:

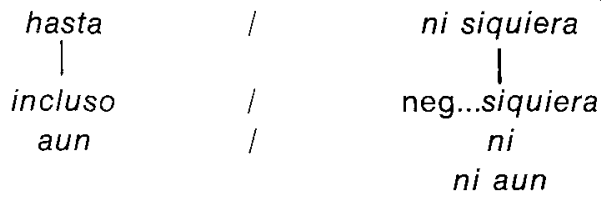

6. Estos adjuntos que hemos visto que están ligados al elemento al que afectan, manifestando un contenido presuposicional, tienen en cierto modo un valor conectivo. Los términos sobre los que versa nuestro estudio presuponen que toda una serie de elementos que forman parte de su paradigma han cumplido positiva o negativamente la predicación. Además, sirven de engarce en la conversación, ya sea directamente apuntando hacia un contexto lingüístico, más o menos cercano (lo menos habitual), o a una realidad extralingüística que forma parte de la comunicación. Ese valor no lo tenía, por ejemplo, el adverbio bien que analizamos. Pero sí los enlaces conjuntivos, que establecen esa conexión pero de manera discursiva: relacionando enunciados (sobre este punto trabajamos actualmente). Así, incluso, como vimos $(/ 13 /)$.

Los adjuntos que estudiamos, por el contrario, pueden ir referidos a cualquier elemento de cualquier categoría, de cualquier función:

- Sintagmas nominales sujetos:

/32a/: "Hasta los animales se asustaron"

$/ 32 \mathrm{~b} /$ : «Ni siquiera los animales se asustaron"

132c/: «Incluso los animales se asustaron"

- Sintagmas verbales:

/33a/: «No sólo bebi, sino que hasta me emborraché"

/33b/: «No sólo no me emborraché, sino que ni siquiera bebí»

$133 \mathrm{c} /$ : "No sólo bebí, sino que incluso me emborraché»

- Segmentos en función adverbial, ya sea de construcción nominal: 134/: "Hasta por mi enemigo debo luchar"

135/: «Ni siquiera por los amigos debe uno preocuparse»

|36/: "Incluso en la cocina hace calor»

u oracional:

137/: "Hasta donde vive hace frío"

138/: «Ni siquiera cuando trabaja se rie»

139/: "Incluso cuando está con los amigos está triste»

- También con adjetivos:

140/: "Se pone hasta bonita"

141/: «No está ni siquiera alegre, cuanto más borracha»

142/: «Habla incluso altanera»

Sin embargo, esto cambia, según $R$. Cano, cuando se refiere a un sintagma verbal, como ya expusimos al principio. Si recordamos, nos decía que se aplicaba a todo el predicado, menos a los 'adjuntos' (complementos) locativos y temporales. Interpretamos esto en el sentido de que se aplica a dichos elementos cuando son participantes fundamentales de la frase, no cuando son marginales de los cuales puede prescindirse. Por ejemplo: 
143/: «Hasta $\rightarrow$ / llegamos a Barcelona / »

Incide sobre todo el predicado, porque a Barcelona forma parte de las valencias del verbo. $Y$ :

/44/: «Juan y Antonio hasta $\rightarrow$ / se sentaron encima de la mesa / » Pero:

145/: «Hasta $\rightarrow$ / llovió / a las 12»

/46/: «Hasta $\rightarrow /$ nos citamos a las $12 / »$

$Y$ también señala que lo importante en este contexto (ante un sintagma verbal) es la noción 'nuevo' o 'rema', junto con la de énfasis: afecta a lo nuevo, no a la información dada. De ahí que en las siguientes frases, que él mismo pone:

/47a/: "Hasta $\rightarrow$ / quemaron coches / "

/47b/: «Hasta $\rightarrow$ / quemaron / los coches",

«enfatice» a todo el sintagma verbal en la primera, porque es información nueva todo, no es conocido el implemento. En la segunda está presentado, es conocido, y, en consecuencia, influye sólo en el verbo.

Sin embargo, puede aparecer el contraejemplo:

148/: «Hasta $\rightarrow$ / nos habíamos comprado los libros / cuando cerró el colegio» Creo que presupone que habíamos hecho algunas cosas y hasta esa: comprar los libros.

Puede afectar al verbo, pues, o al verbo y el implemento, o a toda la oración.

¿Se trata, entonces, de elementos rematizadores o de enfatizadores? La primera posición es la que goza de más adeptos. Entre ellos Bosque, H. Contreras (Contreras, 1978: 132) e incluso C. Rojas Nieto que los trata como expansiones de la coordinación (Rojas Nieto, 1982: 133-147). R. Cano habla, como hemos visto, de énfasis, aunque mezcla este criterio con el de rema al tocar los casos en que hasta afecta al sintagma verbal.

Del ejemplo anterior también deducimos que a veces no tiene tanta importancia que el elemento (el implemento sobre todo) aparezca determinado o no. En la oración /48/ lo que resalta el hablante es la acción de comprar los libros que se presenta como nueva para el oyente, independientemente de que los libros sea el elemento conocido, ya que está determinado culturalmente o por la situación empírica o práctica.

Lo que hay que tener en cuenta es que con estos elementos, hasta, incluso y $n i$ siquiera, el hablante saca un miembro de una serie, elemento que considera importante y que cree que el oyente no conoce y al que se presupone le va a sorprender. Esto significa que para el hablante ese elemento es el no conocido, el que se presenta como más informativo de la frase, o, al menos, es lo que dicho hablante cree. Es un elemento remático o una «información nueva», como prefiere llamarla $H$. Contreras. No nueva en el sentido de que no conozcamos la existencia de esos elementos en la realidad, sino nueva en este contexto. En este sentido hay que entender lo que decía $R$. Cano de que lo nuevo era la introducción de un elemento semántico en una posición sintáctica.

Pero además de esto, estos adverbios se diferencian de cualquier otro procedimiento rematizador en que el hablante tiene unas expectativas con respecto a la actitud del oyente. Presupone que el oyente no esperaba su aparición en el discurso, ya que son los elementos que pragmáticamente ocuparían el escalón más bajo en la escala de probabilidad que el hablante supone que el oyente tiene. Esa 
sorpresa, esa condición de ser «elemento no esperado» es lo que provoca su énfasis.

Por lo tanto, estos adjuntos son procedimientos remáticos aunque de las presuposiciones que reelaboran surja como consecuencia pragmática una enfatización del elemento presentado.

Hay, pues, que distinguir la pragmática de lo que pueden ser procedimientos sintácticos.

7. En conclusión, estos elementos considerados aquí se organizan en virtud de un eje: positivo / negativo. Entre los positivos tenemos hasta e incluso (aunque este último puede no ser sólo adjunto, sino también enlace conjuntivo). Y en el negativo ni siquiera con sus variantes formales. Diríamos, entonces, que el elemento sería la fórmula «negación+siquiera».

En consecuencia, la pareja polar seria hasta / ni siquiera. Ambos sólo pueden ser adjuntos y no pueden usarse en contextos que no sean el afirmativo o el negativo respectivamente. Incluso es un elemento que puede tener el mismo valor presuposicional que hasta aunque con mayor movilidad sintáctica. Y puede ser compatible con la negación también. Sólo hasta es fijo, no móvil, herencia, quizás, de su origen prepositivo.

El sentido en que los hemos estudiado aquí es en su comportamiento como adjuntos introduciendo y afectando a un segmento funcional cualquiera.

Ahora bien, ¿a qué categoría pertenecen dichos elementos? Algunos los llamaban conjunciones, como Lenz, que considera hasta una conjunción copulativa de gradación (Lenz, 1935: 549). Para Bello era uno de esos elementos «que saliendo de su uso primitivo se transforman en meros afijos" (Bello-Cuervo, 1970: 1.246). El diccionario de la Academia, Sánchez Márquez (1982) y otros la consideraban «conjunción copulativa, y entonces sirve para exagerar o ponderar una cosa, y equivale a también y aun» (R.A.E., 1984: 721).

Para el Esbozo (R.A.E., 1983) es una partícula incluyente. Y C. Corrales (1978) la considera partícula enfática, ya que se puede conmutar por cero sin que se altere el contenido esencial del mensaje. Su presencia o ausencia viene motivada por aspectos de intensidad expresiva. En este contexto, «pertenece al mismo paradigma que incluso, aun y también, habitualmente adverbios» (Corrales, 1978: 68).

$J$. Feuillet considera que esta no es una categoria ya que hay elementos muy distintos en ella y según él, el adverbio «devrait être uniquement réservé au groupe invariant en fonction circonstancielle» (Feuillet, 1981: 27). El elemento correspondiente a los estudiados aqui entraría dentro de una clase que él llama "sublocutifs": «Les unités qui sont chargées d'apporter la marque du locuteur dans l'énoncé (jugement sur le contenu propositionnel, émotions, opérations logiques, etc.)" (Feuillet, 1981: 22). Dentro de ese grupo se reconocen tres subtipos: «modalisateurs» ( $p$. 24), «énonciateurs» (p. 25) y «modificateurs» (p. 26). En este último, en la subclase de los intensivos, se incluyen.

R. Cano las llama partículas, ya que en concreto las que nosotros estudiamos no cumplen la función terciaria definida para el adverbio: «no modifican el contenido léxico del adjetivo o adverbio que pueden acompañar» (Cano, 1982: 251). De la misma manera Bello las llama cuasiafijos «porque se anteponen a toda clase de 
palabras modificando su significado y sirviendo como de partículas prepositivas" (Cano, 1982: 251).

Sin embargo, P. Carbonero (1978) partiendo del mismo principio de los rangos, llega a conclusiones distintas a las de Cano: «Teniendo en cuenta el concepto de «rangos» de $O$. Jespersen, podríamos afirmar que el rasgo funcional básico del adverbio es el de ser categoría terciaria" (Carbonero, 1978: 181). Así, tomándolo en un sentido amplio "será terciario aquello que por exclusión no sea ni primario ni secundario; ni el sustantivo, no lo que incide sobre el sustantivo. Por ello, hablar de jerarquia terciaria es hablar de una categoria que funciona como incidencia de incidencias" (Carbonero, 1978: 182, y cfr. Karcevski, 1936: 107).

Pero aun así no queda completamente definida la función sintáctica de estos elementos, ya que hay unidades que se aplican no sobre un elemento concreto, sino que "suponen una incidencia 'englobadora' sobre construcciones de nivel superior, como son los sintagmas u oraciones" (Carbonero, 1978: 182). Así pues, de esa «incidencia de incidencias» surgen todas las siguientes posibilidades funcionales 0 incidencias sintácticas:

«a) Sobre verbo o equivalente.

b) Sobre adjetivo o equivalente.

c) Sobre alguno de los otros dos a) o b).

d) Englobadora de sintagmas nominales.

e) Englobadora de sintagmas verbales u oraciones" (Carbonero, 1978: 183). Todas estas incidencias, aunque formuladas de manera distinta, le reconoce $\mathrm{E}$. Buyssens (1975). Pero, además, para él es una categoría de por sí, ya que a diferencia del resto de ellas, "tous les adverbes ont toujours la fonction de complément et qu'ils se distinguent des noms et des pronoms - qui peuvent aussi être complément- par le fait qu'ils ne peuvent jamais avoir un adjectif sous leur dépendance» (Buyssens, 1975: 461).

De acuerdo con lo expuesto por Carbonero, hasta, incluso y ni siquiera son elementos que tienen todas esas posibilidades combinatorias.

Ahora bien, no se trata de una modificación sintáctica. Los adverbios hasta, incluso o ni siquiera modifican no a un sintagma nominal, sino a la relación predicativa. En

149/: «Hasta los niños vinieron»

no recae su incidencia en los niños, sino en la relación los niños-venir. Se trata de elementos que desempeñan su misión en el nivel textual. Sirven para rematizar un participante de la oración. Si queremos situarlo a nivel oracional, modificarían a la relación predicativa, o, para aclararnos más, a su representante nuclear: el verbo.

Quede claro, pues, que pertenecen a otra dimensión no tratada hasta ahora por quedarse las gramáticas ceñidas a los márgenes de la oración. De ahí la ambigüedad y confusión que reina en este campo. Su dominio de acción es otro: el textual. En esta dimensión se sitúan todos estos elementos cuya misión no es la de la modificación sintáctica, sino que establecen una relación presuposicional con el contexto o la realidad.

En términos de gramática de la oración, se incluirian en la categoría adverbio. Hay, pues, entre estos elementos, una serie de ellos que afectan a un sintagma añadiéndole ciertas características pragmáticas o presuposicionales que establecen una cohesión comunicativa. Su contenido no es pleno como el de los más tradicio- 
nales de este grupo. Pero son elementos que es necesario estudiar, ya que constituyen un paradigma de formas muy usadas en la comunicación. Fundamentalmente hemos usado un análisis de aserción-presuposición que creemos revela el verdadero contenido de ellos. Queda abierto, pues, el análisis de los adjuntos, una función de los adverbios de la que sólo se trata normalmente un grupo: los más libres o los de contenido semántico pleno, pero no los más ligados o de contenido no puramente léxico, sino de conexión con la realidad extralingüística.

Hay que ampliar las miras para acoger a todos estos elementos que desempeñan su misión dentro del discurso, del texto. En este dominio textual la pragmática, como hemos visto, tiene mucho que decir.

\section{Referencias bibliográficas}

Alarcos Llorach, E. (1984), Estudios de gramática funcional del español, 3. a edición, Madrid, Gredos.

Alcina, J.; Blecua, J. M. (1983), Gramática española, 4. ${ }^{a}$ edición, Barcelona, Ariel. Allerton, D. J.: Cruttenden, A. (1974), «English Sentence Adverbials: Their syntax and their intonation in British English", Lingua, 34, pp. 1-30.

Anderson, S. R. (1972), "How to get even", Language, 48, pp. 893-906.

Barrenechea, A. M. ${ }^{2}$ (1979), "A propósito de la elipsis en la coordinación», en Aa. Vv. Estudios lingüísticos y dialectológicos - temas hispánicos, Argentina, Hachette, pp. 21-37.

Bello, A.; Cuervo, R. J. (1970), Gramática de la lengua castellana, 8. edición, Buenos Aires, Sopena.

Bosque, I. (1980), Sobre la negación, Madrid, Cátedra.

Buyssens, E. (1975), «La classification des adverbes", Revue Roumaine de Linguistique, XX, 5, pp. 461-463.

Cano, R. (1982), "Sujeto con preposición en español y cuestiones conexas», Revista de Filologia Española, LXII, pp. 211-258.

Carbonero, P. (1978), "Criterios para una caracterización funcional de los adverbios", Revista de la Sociedad Española de Lingüistica, 8, pp. 169-197.

Carratalá, E. (1980), Morfosintaxis del castellano actual, Barcelona, Labor.

Contreras, H. (1978), El orden de palabras en español, Madrid, Cátedra.

Corrales, C. (1978), «Sobre el sujeto con preposición», Estudios ofrecidos a E. Alarcos Llorach, t. II, Oviedo, pp. 65-77.

Domínguez de Rodríguez-Pasqués, P. (1970), «Morfología y sintaxis del adverbio en -mente», Actas del III Congreso Internacional de Hispanistas, Méjico, pp. 293303.

Echaide, A. M. ${ }^{a}$ (1974), «La coordinación adversativa en español: aspecto sincrónico", Revista de Filología Española, LVII, pp. 1-33.

Fauconnier, G. (1975a), «Pragmatic Scales and Logical Structure», Linguistic Inquiry, 6 , pp. 353-375.

- (1975b), "Polarity and the Scale Principle», Papers from the 11th. Regional Meeting Chicago Linguistic Society, pp. 188-199.

Feuillet, J. (1981), «Peut-on parler d'une classe de l'adverbe?», La Linguistique, 17, pp. $19-27$. 
Fraser, B. (1971), "A analysis of 'even ' in English", Fillmore, Charles J. Langendoen, D. Terence (eds.), Studies in Linguistic Semantics, Holt, Rinehart \& Winston, pp. $150-178$.

Greenbaum, S. (1970), Studies in English Adverbial Usage, London, Longman.

Horn, L. R. (1969), "A presupositional analysis of only and even», Papers from the 5th. Regional Meeting Chicago Linguistic Society, pp. 98-107.

Karcevski, S. (1936), "Sur la nature de l'adverbe», Travaux du Cercle Linguistique de Prague, VI, pp. 107-111.

Lenz, R. (1935), La oración y sus partes, 3. a edición, Madrid, Publicaciones de la Revista de Filología Española.

Martin, R. (1974), “La notion d'«adverbe de phrase»: essai d'interpretation en grammaire générative», Rohrer, Christian-Ruwet, Nicolas (eds.), Actes du CoIloque Franco-Allemand de Grammaire Transformationnelle, Tübingen, Niemeyer, pp. 66-75.

Martínez Amador, E. M. (1966), Diccionario gramatical y de dudas del idioma, Barcelona, Sopena.

Moliner, M. ${ }^{a}$ (1983), Diccionario de uso del español, t. I, Madrid, Gredos.

Real Academia Española (1983), Esbozo de una nueva gramática de la lengua española, Madrid, Espasa-Calpe.

- (1984), Diccionario de la lengua española, 20. a edición, Madrid, EspasaCalpe.

Rivero, M. ${ }^{a}$ L. (1977), Estudios de gramática generativa del español, Madrid, Cátedra.

Rojas Nieto, Cecilia (1982), Las construcciones coordinadas sindéticas en el español hablado culto de la ciudad de México, México, Universidad Nacional Autónoma de México.

Sánchez Márquez, M. J. (1982), Gramática moderna del español, 2. ${ }^{a}$ edición, BarceIona, Ediar.

Schreiber, P. A. (1971), «Some Constraints on the formation of English sentence adverbs", Linguistic Inquiry, 2, pp. 83-101.

Seco, M. (1967), Diccionario de dudas de la lengua española, $5 .^{a}$ edición, Madrid, Aguilar.

Shanon, B. (1978), «Even, only and almost hardly», Studies in Language, 2, pp. 35-70. 